\title{
Erratum to: OpenHELP (Heidelberg laparoscopy phantom): development of an open-source surgical evaluation and training tool
}

\author{
H. G. Kenngott ${ }^{1}$ J. J. Wünscher ${ }^{1} \cdot$ M. Wagner ${ }^{1} \cdot$ A. Preukschas ${ }^{1}$ \\ A. L. Wekerle ${ }^{1} \cdot$ P. Neher ${ }^{2} \cdot$ S. Suwelack ${ }^{3} \cdot$ S. Speidel ${ }^{3}$. \\ F. Nickel ${ }^{1} \cdot$ D. Oladokun ${ }^{1} \cdot$ Lorenzo Albala $^{4} \cdot$ L. Maier-Hein ${ }^{2}$. \\ R. Dillmann ${ }^{3}$ - H. P. Meinzer ${ }^{2}$ - B. P. Müller-Stich ${ }^{1}$
}

Published online: 14 July 2015

(C) Springer Science+Business Media New York 2015

\section{Erratum to: Surg Endosc}

DOI 10.1007/s00464-015-4094-0

Lorenzo Albala, Ruprecht-Karls-University, Department of General Abdominal and Transplant Surgery, University of Heidelberg, Im Neuenheimer Feld 110, 69120 Heidelberg, Germany, was inadvertently omitted as the eleventh author.

The online version of the original article can be found under doi:10.1007/s00464-015-4094-0.

B. P. Müller-Stich

Beat.Mueller@med.uni-heidelberg.de

1 Department of General, Visceral and Transplantation Surgery, University of Heidelberg, Im Neuenheimer Feld 110, 69120 Heidelberg, Germany

2 Division of Medical and Biological Informatics, German Cancer Research Center (DKFZ), Heidelberg, Germany

3 Institute for Anthropomatics, Karlsruhe Institute of Technology, Karlsruhe, Germany

4 Department of General Abdominal and Transplant Surgery, Ruprecht-Karls-University, University of Heidelberg, Im Neuenheimer Feld 110, 69120 Heidelberg, Germany 